・ 《生物多样性公约》履约专题・

\title{
重视女性在生物多样性保护中的作用
}

\author{
刘海鸥 ${ }^{1,2}$ 陈海君 ${ }^{2}$ 刘 蕾 ${ }^{2}$ 晏 薇 ${ }^{2}$ 薛达元 ${ }^{1 *}$ \\ 1 (中央民族大学生命与环境科学学院, 北京 100081) \\ 2 (环境保护部环境保护对外合作中心全球环境基金/绿色气候基金项目执行办公室，北京 100035)
}

\begin{abstract}
摘要: 中国是生物多样性大国, 也是高度重视性别平等的国家之一, 在履行《生物多样性公约》和世界性别平等 舞台上均扮演着重要角色。但目前中国在生物多样性保护中的性别主流化方面仍进展较慢, 这与其在推进生物多 样性保护方面发挥的积极作用不相匹配。为此, 本文通过梳理生物多样性与性别的特别关系, 探讨了生物多样性 保护中性别概念的产生和演化过程，分析了国际组织、多边基金和一些国家在生物多样性保护中性别主流化方面 的研究成果和工作进展, 进而提出加强性别与生物多样性问题的研究、相关机构能力的建设、性别与生物多样性 的政策导入以及国内生物多样性项目的管理等建议, 以期为中国在未来生物多样性与性别领域开展工作、国家履 约和参与《生物多样性公约》国际谈判提供参考。
\end{abstract}

关键词：生物多样性; 生物多样性公约; 性别平等; 性别主流化

\section{Incorporating gender equality into biodiversity conservation}

\author{
Haiou Liu ${ }^{1,2}$, Haijun Chen², Lei Liu², Wei Yan², Dayuan Xue ${ }^{1 *}$ \\ 1 College of Life and Environmental Sciences, Minzu University of China, Beijing 100081 \\ 2 GEF/GCF Implementation Office, Foreign Economic Cooperation Office, Ministry of Environmental Protection, Bei- \\ jing 100035
}

\begin{abstract}
China is rich in biological diversity resources and attaches great importance to gender equality as a country. China plays an important role in the worldwide realm of the implementation of the Convention on Biological Diversity (CBD) and gender equality. However, gender mainstreaming in biodiversity conservation in China is still in its early stages, and does not match with positive roles promoting biodiversity conservation and gender equality nationally. Therefore, we analyze the special relationship between biodiversity and gender, discuss the generation and evolution of the concept of gender in biodiversity conservation, and summarize the progress of international organizations, multilateral funds and frontrunner nations regarding gender mainstreaming in biodiversity conservation. We put forward suggestions, including strengthening research on gender and biodiversity issues, enhancing capacity-building of related agencies, promoting the introduction of gender and biodiversity issues into policy decision-making, and improving domestic biodiversity project management, all with the aim to provide guidance for follow-up work and a reference for the implementation and negotiation of the Convention on Biological Diversity in China.
\end{abstract}

Key words: biodiversity; Convention on Biological Diversity; gender equality; gender mainstreaming

近年来, 生态系统功能不断退化、物种濒危程 度加剧、遗传资源不断丧失和流失等现象引起了国 际社会对生物多样性问题的极大关注(环境保护部, 2011), 保护生物多样性成为国际社会的普遍共识。 在生物多样性保护工作中推动性别主流化近年来
得到了国际社会的广泛关注，“生物多样性与性别” 已经作为热点议题写入 《生物多样性公约》 (https://www.cbd.int/gender/)。但在生物多样性保护 过程中, 脱离社会性别因素孤立地考虑生物多样性 问题是片面的，原因在于女性和男性在生物多样性

收稿日期: 2017-09-01; 接受日期: 2017-11-16

基金项目: 中央民族大学高等学校学科创新引智计划(B08044)

* 通讯作者 Author for correspondence. E-mail: xuedayuan@hotmail.com 
资源的保护和利用等方面存在显著的性别差异 (https://www.cbd.int/gender/doc/fs-gender-perspective s-en.pdf/)。全面认识和探讨性别与生物多样性之间 的关联性, 将性别因素纳入保护生物多样性的各个 方面, 对于推进性别平等、推动生物多样性资源的 保护、可持续利用以及遗传资源获取与惠益共享、 实现绿色发展和生态文明建设具有重要意义。

\section{1 性别与生物多样性的关系}

众多案例和资料显示, 女性和男性对于生物多 样性有着不同的使用方式、知识和实践。根据 Huisinga等(1993)的研究, 地方传统知识的性别特 点主要表现在 4 个方面: 一是女性和男性均掌握了 对不同事物的知识; 二是女性和男性对于相同事物 所拥有的知识不同; 三是女性和男性可能对他们知 识的认知方向不同; 四是女性和男性采用不同的方 式传递他们的知识。这些区别使女性在生物多样性 保护、可持续利用和遗传资源获取与惠益分享方面 与男性一样发挥着不可替代的作用。

\section{1 女性在生物多样性保护方面的作用}

女性作为地方社区的守护者、农业生产的主力 军、农村家庭的管理者和农业文化的传承者, 在保 护生物多样性方面扮演着重要角色(蒋爱群和冯英 利, 2011)。

\subsection{1 女性在植物种质资源多样性保护方面的作用}

在很多国家, 女性都负责农作物和蔬菜种子的 选择、保存和管理。安第斯山区的女性基于其烹饪 喜好选择土豆品种(Sillitoe \& Howard, 1981), 卢旺 达女性种植了 600 多种豆类植物, 秘鲁阿瓜鲁纳族 地区女性种植了60多种薯类(http://www.fao.org/sd/ 2001/PE1201a_en.htm/), 在美国宾夕法尼亚和秘鲁 安第斯山区, 女性在保存种子以及维持遗传多样性 方面发挥着主要作用(Sachs, 1997)。墨西哥女性在 农业生产中通过利用粮食作物和药用植物提升了 当地的植物多样性。这些知识和管理使当地社区得 到持续发展(Cabrera et al, 2001)。

除了广泛种植的品种以外, 女性还对一些较少 利用的植物的种植和保存发挥着重要作用, 这些植 物一般仅在特定社区作为补充食材。例如, 在也门, 一些农作物仅由女性种植, 被称为“女性作物”, 包 括花生、南瓜、药用植物、哣豆、黄瓜和红薯等。 这些农作物对提高农场生物多样性和粮食安全产 生了积极影响(https://www.cbd.int/doc/world/ye/yenbsap-01-en.pdf/)。不丹的国家生物多样性战略与行 动计划中指出, 他们已经认识到人们不经常利用的 植物对家庭食物和生计安全也具有重要作用, 而这 些植物的管理和采摘往往由女性负责(https://www. cbd.int/doc/world/bt/bt-nbsap-v2-p1-en.pdf/)。类似知 识并非仅局限在食用植物方面, 在马里, 由于当地 女性经常使用一些韧性较强的植物品种制作篮子 等容器而使得这些植物具有了特殊价值(https:// www.cbd.int/doc/world/ml/ml-nbsap-01-p1-fr.pdf/)。可 见, 女性在植物种质资源多样性保护方面发挥了不 可替代的作用。

\subsection{2 女性在家畜种质资源多样性保护方面的作用}

妇女在畜牧业生产经营、草地生态保护与建设 以及家庭事务管理等方面扮演着更加重要的角色 (Zhang \& Zhang, 1994)。妇女喂养牲畜、饲养家禽, 如羊、兔和猪等(Shen \& Qian, 2009)。在中国祁连山 牧区的农村社会变革中, 女性是畜牧业生产的主力 军, 承担着将近 $80 \%$ 的生产管理工作(荷钰姣等, 2015)。在墨西哥东南部，女性在其后院中保存和饲 养着多个地方品种的鸡、鸭等, 并能根据当地环境 选择出最适合的品种(http:www.fao.org/gender/en/ag ri-e.htm/)。可见, 女性在家畜种质资源多样性保护 方面也发挥着重要作用。

\section{2 女性在生物多样性可持续利用方面的作用}

Malika等(2008)对西亚地区包括叙利亚、黎巴 嫩、约旦等国的农业活动和农业生物多样性保护进 行调查发现, 女性关于地方品种属性以及对药用植 物、香料植物的知识对农业生物多样性的保护和可 持续利用起到了重要作用。在一些社会, 女性提供 了超过 $80 \%$ 的野生植物食物。一个针对印度北方邦 的研究发现(https://www.cbd.int/gender/doc/fs_uicn-c bd_abs.pdf/), 男性主要使用与农业生产有关的植物, 女性主要使用与家庭生计有关的植物, 这些植物主 要用作药材、保健品、清洁用品、纤维、食物和工 具; 女性通过森林和公用土地获得的收入占其总收 入的33-45\%, 而男性仅占13\%。通过案例研究发现, 女性在一些物种通过可持续利用得到保护、进而实 现人与自然和谐相处的生活方式中扮演着重要角 色(Martina, 2005)。

\section{3 女性在遗传资源获取与惠益分享中的重要作用} 女性在遗传资源获取与惠益分享方面发挥着 
不可替代的作用。首先, 女性在野生物种、当地植 物品种和种子的篮选和使用方面起着关键作用, 很 大程度上掌握着价值丰富的遗传资源; 其次, 女性 往往负责重要传统知识的掌握、发展和传播(Howard, 2003)。

在一些发展中国家，多达 $80 \%$ 的人口将传统医 药作为其医疗保健的主要手段, 与男性相比, 女性 对药用野生植物的知识往往更多、更专业(Sillitoe \& Howard, 1981; https://www.cbd.int/doc/world/sv/sv-n bsap-01-p1-es.pdf/)。Rodriguez等(2004)对巴西国家 公园的调查显示, 助产师拥有对某些特定植物的知 识; 而在尼加拉瓜的地方社区调查时, 当问及社区 周围和森林中的药用植物种类时, 男性往往倾向于 求助他们的配偶。世界自然保护联盟(International Union for Conservation of Nature, IUCN)在一份报告 中指出, 在遗传资源获取与惠益分享中性别主流化 对于可持续发展和《生物多样性公约》(以下简称《公 约》)目标的实现发挥着极其重要的作用(https://ww w.cbd.int/gender/doc/fs_cbd_biodiversity.pdf/)。

\section{2 生物多样性保护中性别主流化问题的产 生和演化}

“社会性别”一词最早出现在20世纪70年代初 的国际妇女运动中, 80 年代后逐渐被联合国广泛采 用, 是近年来国际社会分析性别平等的基本概念 (刘伯红, 2009)。一些西方国家首先制定了性别平等 的法律和政策, 用以抵制性别歧视, 使性别平等概 念得到巩固和发展。经过几十年的发展, 越来越多 从事性别相关领域研究的机构和个人由关注性别 平等概念演变为更加强调性别主流化的概念(王爱 华和潘寻, 2015)。性别主流化的含义是在追求性别 平等时更多关注社会发展主流的性别敏感问题, 从 而确保社会发展的过程和结果是建立在性别平等 的基础之上, 这就是所谓的社会性别主流化的基本 思想(李小云, 2001)。

关于性别与生物多样性问题的研究始于 20 世 纪80年代, 在国际政策层面始于2005年联合国开发 计划署(United Nations Development Programme, UNDP)的人类发展报告, 报告中首先把消除性别歧 视作为实现千年发展目标的一项重要举措, 并把性 别平等问题和减贫问题进行了关联。此后《公约》 首次正式将性别和生物多样性问题联系起来。随后,
《公约》的科学、技术和工艺咨询附属机构(Subsidiary Body on Scientific, Technical and Technological Advice, SBSTTA)就女性实践、知识、公平展示 和性别角色等问题提出了具体建议, 将生物多样性 与性别问题和减贫问题分别考虑。在上述建议的基 础上, 2007年, 《公约》秘书处进一步提出了在《公 约》和联合国环境规划署(UNEP)的框架内纳入性别 主流化的问题, 从而使生物多样性保护中性别主流 化的概念得到正式确立。

2010年，《公约》秘书处编制了《促进国家生 物多样性保护战略与行动计划中性别主流化指南》, 并在同年召开的第10次缔约方大会的决议X/19中 得到采纳。该指南要求将性别问题纳入到《公约》 的所有工作中, 还要求《公约》秘书处制定明确的 指标以监督性别主流化的实施进展。

目前, “生物多样性与性别”已在《公约》官网上 成为一项独立的主题(https://www.cbd.int/gender/), 并在《公约》的相关决议中做了明确规定。例如，《公 约》在序言中规定, “并认识到妇女在保护和持久使 用生物多样性中发挥的极其重要作用，并确认妇女 必须充分参与保护生物多样性的各级政策的制订 和执行” (https://www.cbd.int/doc/legal/cbd-zh.pdf/); 《公约》决议 $V / 16$ 条 [关于第 $8(j)$ 款及相关条款]规 定, “认识到女性在生物多样性保护和可持续利用 中扮演的重要角色，强调应当对这种角色予以强 化, 对土著和地方社区女性参与规划工作给以更 多关注”。《公约》相关工作组的工作计划也对性别 问题进行了规定, 为生物多样性的性别主流化工 作提供了法律依据。

\section{3 生物多样性保护中性别主流化的做法和 进展}

\section{1 生物多样性相关国际组织的做法}

性别与生物多样性主题的产生和发展以及 《公 约》中性别议题的确立等工作都与国际组织的推动 息息相关，尤其是世界银行、亚洲开发银行、联合 国开发计划署和IUCN等机构。

世界银行和亚洲开发银行在国际规则制定方 面的影响深远而且广泛。世界银行将人类发展与性 别作为一项重要主题, 在该主题下设立了社会性别 的分主题, 介绍其性别研究的最新进展、相关工具 和做法, 并依靠其广泛的影响力推动了性别政策在 
全球多个地区的实施，如非洲、东亚太平洋地区、 欧洲和中亚、拉美和加勒比海地区、中东和北非以 及南亚等地区 (http://www.worldbank.org/en/topic/gen der/overview/)。亚洲开发银行的做法与世界银行类 似，也制定了性别政策、原则和指南，设立了性别与 发展的主题, 通过贷赠款项目推动其性别战略和政 策的实施(http://www.adb.org/themes/gender/main/)。

IUCN的做法与世界银行和亚洲开发银行不同, 主要是通过对环境类国际公约的影响来推行其制 定的性别原则、政策、目标和指标体系。IUCN推 动了包括《公约》在内的多个国际公约性别主题和 议题的确立, 并制作了多套与性别和环境相关的宣 传材料在相关国际会议、活动中散发, 包括“性别与 生物多样性”、“荒漠化公约性别政策框架”、“将性 别融入里约三公约”、“将性别融入全球环境基金”、 “美国国际开发署宣传材料”等。

在项目执行实施层面, 联合国开发计划署将性 别平等和给予女性权利作为其主要使命, 并确立了 “性别与可持续发展”、“性别、气候与灾难响应”等 主题, 通过执行和实施的国际项目, 从政策、项目、 宣传等多个层面和角度推动性别主流化 (http://ww w.undp.org/content/undp/en/home/ourwork/gender-eq uality/overview.html/)。联合国粮农组织(Food and Agriculture Organization of the United Nations, FAO) 则聚焦农业生物多样性, 对性别与农业生物多样性 问题进行了大量调查和分析, 为相关问题提供了理 论和数据支持(http://www.fao.org/sustainable-develop ment-goals/en/)。

\section{2 多边基金的做法}

受到IUCN、世界银行等国际组织和环境国际 公约的影响，《公约》的唯一资金机制“全球环境基 金” (GEF) 将性别政策作为其重要的管理政策, 并 在机构治理、项目管理等层面推动这些政策的实施, 取得了良好效果。2011年, GEF发布了性别主流化政 策, 并通过贷赠款项目进行推进。在项目设计、实 施、监测与评估、验收等各个环节全程考虑性别因 素。如GEF在其项目申报模板中, 设计了专门的性 别分析模块, 确保项目申请机构在项目设计阶段充 分考虑性别平等因素; 在项目实施阶段, GEF委托 执行机构对相关指标进行监测和评估, 确保项目中 与性别有关的活动能够按照计划开展; 在项目验收 阶段, 对设定的目标完成情况和质量进行考核, 并
依靠GEF平台传播成功经验和案例(http://www.thegef.org/publications/gender-equality-action-plan/)。

与GEF类似, 2015年, 专门服务于《联合国气候 变化框架公约》的绿色气候基金在其第九次董事会 上通过了 “绿色气候基金性别政策”, 并同时发布了 《2015-2017年性别行动计划》, 依托贷赠款项目在 设计、执行和实施各个层面推动性别政策落地。

\section{3 部分国家的做法}

在《公约》的影响下, 一些国家已经开始按照 《公约》关于性别的要求, 将性别主流化问题纳入 国家生物多样性战略与行动计划 (National Biodiversity Strategy and Action Plan, NBSAP)。2008年4 月, IUCN调查的 160 个当时已经编制了国家生物多 样性战略与行动计划的国家中, 有77个提到了性别 或女性问题。比如, 墨西哥是《公约》支持的 3 个试 点国家之一, 已明确将性别问题纳入国家生物多样 性政策、计划和规划中, 并承诺将性别主流化落实 到生物多样性保护行动中。乌干达也是《公约》的 试点国家, 采用了与墨西哥类似的做法(https:// www.cbd.int/nbsap/search/)。

\section{4 中国如何在保护生物多样性中推进性别 主流化}

目前中国学者对相关领域的研究主要聚焦于 女性劳动力与农业发展的角度(李旻和彭艳斌, 2009; 吴慧芳和饶静, 2009; 刘䈗红和姚德超, 2012), 仅有少数研究涉及了女性与农业生物多样 性问题(蒋爱群和冯英利, 2011)。此外, 中国国家生 物多样性战略与行动计划几乎没有涉及性别问题, 相关研究也几近于空白。目前存在的问题主要表现 在: 缺少相关研究和理论依据作为支撑; 国内生物 多样性相关机构在性别主流化方面能力不足; 生物 多样性相关政策中没有考虑性别因素; 生物多样性 项目中缺少对性别因素的考核指标。基于上述问题 和挑战, 本文提出如下建议:

(1)加强性别与生物多样性问题研究, 为相关 工作的开展提供理论支撑。首先, 应考虑将性别分 析纳入研究方法体系。通过梳理女性和男性在生物 多样性相关问题中的不同表现, 帮助研究人员探索 女性和男性在生物多样性项目中的不同角色和关 系, 如女性和男性如何获取和使用生物多样性资源, 他们分别拥有哪些不同种类的知识等。然后, 初步 
识别生物多样性研究中的社会性别差异, 收集性别 分类数据, 这可以通过问卷调查、参与式评价等方 法来实现。调查的问题可以包括: 女性和男性负责 哪些不同的活动? 获取和使用哪些资源? 参与家 庭和社区决策的情况、日常喜好等。再次, 确定研 究的优先顺序, 找出重要的性别差异, 并根据这些 差异调整研究方法。最后, 基于上述研究最终确定 性别差异的重要意义。这些研究成果将有益于保护 中国传统知识, 尤其是女性在生物多样性保护和可 持续利用过程中的经验, 为生物多样性相关法规政 策的制定提供依据。

(2)加强相关机构能力建设, 服务国家“一带一 路”和“走出去”战略。生物多样性与性别在国际上属 于热点前沿问题, 但目前国内生物多样性研究领域 对此关注不够，存在机制不完善、意识较弱等问题， 所以, 应全面提升相关机构的能力建设。首先, 可 以参考一些国家和机构的做法, 选聘具有专业知识 的性别联络人, 专门负责在生物多样性保护中全面 纳入社会性别因素。其次, 在生物多样性管理部门 和机构开展性别主流化培训, 提升工作人员的基本 意识。最后, 有条件的机构可以效仿国际组织的做 法, 制定性别主流化政策, 从机制上解决机构性别 主流化问题。这些做法也将有利于国家“一带一路” 和“走出去” 战略的实施, 减少中国在国外的投资企 业因环境与社会安全保障和性别问题无法满足国 际标准和当地要求的情况。

(3)加强性别与生物多样性政策导向, 服务政 府履约工作。中国是最早加入 《公约》的国家之一, 一直高度重视履约工作(薛达元等, 2012)。在国家层 面, 虽然 2010 年国务院批准实施的《中国生物多样 性保护战略与行动计划(2011-2030 年)》未能强调性 别主流化问题(环境保护部, 2011), 但是在未来国家 生物多样性政策修订和制定方面, 可将性别平等的 因素考虑在内并得以体现; 在地方和部门层面, 应 在地方生物多样性政策中纳入性别平等因素, 有条 件的部门和省(直辖市、自治区)也可以先行试点, 为 国家层面和其他地区相关政策的制定提供示范, 为 我国履行《公约》和参与国际谈判提供支持。

(4)加强国内生物多样性项目管理, 全过程引 入性别平等考核标准。国内纳入了性别平等因素的 大都为国际贷赠款项目，这些项目采用世界银行、 联合国开发计划署等国际机构的性别政策和标准,
为国内机构实现生物多样性项目中的性别主流化 积累了一定的经验。但是, 国内资助的生物多样性 工程和项目对性别平等方面的考虑相对较少。国内 项目管理可以借鉴国际贷赠款项目的做法, 在项目 设计、实施、中期评估和终期验收评估等各个阶段 将性别平等纳入考核。例如, 在项目设计阶段, 需 要制定完整的女性参与方法, 必要时应制定性别发 展计划; 项目实施阶段, 对女性参与项目的情况进 行监测和评估; 在项目验收阶段, 对女性参与的情 况进行终期评估, 总结相关经验, 将好的经验和案 例推广宣传。

\section{参考文献}

Cabrera IR, Martelo EZ, Garcia VV (2001) Gender, rural households, and biodiversity in native Mexico. Agriculture and Human Values, 18, 85-93.

Gou YJ, Liu XY, Zhang WM, Xu CL, Long RJ (2015) An investigation of women's social status and their recognition of the grassland policies in the pastoral area of the Qilian Mountains. Acta Ecologica Sinica, 35, 3472-3479. (in Chinese with English abstract) [苟钰姣, 刘兴元, 张伟明, 徐 长林, 龙瑞军 (2015) 祁连山牧区妇女社会地位及其对 草地政策的认知度. 生态学报, 35, 3472-3479.]

Howard PL (2003) Women and Plants: Gender Relations in Biodiversity Conservation and Management, pp. 131-145. Zed Books, London.

Huisinga NR, Yoder R, Martin Y (1993) Indigenous Agricultural Knowledge and Gender Issues in Third World Agricultural Development, pp. 10-11. Agricultural and International Development, Davis.

Jiang AQ, Feng YL (2011) Roles of rural women in agrobiodiversity conservation and solutions to address their difficulties. Journal of China Agricultural University (Social Sciences Edition), 28(4), 64-70. [蒋爱群, 冯英利 (2011) 农 村妇女在保护农业生物多样性中的作用、困境与出路. 中 国农业大学学报(社会科学版), 28(4), 64-70.]

Liu BH (2009) The concept and features of social gender mainstream. Journal of Shandong Branch, China Female College, 6(1), 1-8. (in Chinese with English abstract) [刘伯红 (2009) 社会性别主流化的概念和特点. 中华女子学院山 东分院学报, 6(1), 1-8.]

Li M, Peng YB (2009) “Feminization” phenomenon of agricultural labor force and its influence on agricultural production-based on the analysis of Liaoning Province. Chinese Rural Economy, 5(1), 61-69. (in Chinese) [李旻, 彭艳斌 (2009) “农业女性化”现象及其对农业生产的影响一一基 于辽宁省的实证分析. 中国农村经济, 5(1), 61-69.]

Liu XH, Yao DC (2012) An analysis of the feminization of agriculture and its formation mechanism. Journal of Hunan 
University of Science \& Technology (Social Science Edition), 4(1), 99-102. (in Chinese with English abstract) [刘筕 红, 姚德超 (2012) 农业女性化现象其形成机制分析. 湖 南科技大学学报(社会科学版), 4(1), 99-102.]

Li XY (2001) What is gender mainstreaming? Chinese Women's Movement, 10(1), 1-5. (in Chinese) [李小云 (2001) 什么是社会性别主流化. 中国妇运, 10(1), 1-5.]

Malika AM, Ahmed A, Mohammed A, Raghed A, Younes S, Ali K (2008) Gender dimension in the conservation and sustainable use of agro-biodiversity in West Asia. The Journal of Socio-Economics, 37(1), 265-383.

Martina AP (2005) Institutional innovations towards gender equity in agrobiodiversity management: collective action in Kerala, South India, pp. 6-43. International Food Policy Research Institute, Washington, DC.

Ministry of Environmental Protection (2011) China Biodiversity Conservation Strategy and Action Plan, pp. 1-34. China Environmental Science Press, Beijing. (in Chinese) [环境保 护部 (2011) 中国生物多样性保护战略与行动计划, pp. 1-34. 中国环境科学出版社. 北京.]

Shen SC, Qian J (2009) Livestock Projects in Southwest, pp. 18-20. LEISA India, Bangalore.

Rodriguez G, Blanco M, Azofeifa F, Jose S, Rica C (2004) Diversity Makes the Difference, pp. 2-5. IUCN, Switzerland.

Sachs CE (1997) Women Working in the Environment Resourceful Natures, pp. 117-119. Routledge, London.
Sillitoe P, Howard PL (1981) The gender of crops in the Papua New Guinea Highlands, pp. 1-14. University of Pittsburgh, Pennsylvania.

Wang AH, Pan X (2015) How to advance gender mainstreaming in environmental projects. Journal of Beijing Forestry University (Social Sciences), 14(4), 17-22. (in Chinese with English abstract) [王爱华, 潘寻 (2015) 如何在环境保护 类项目中推进性别主流化. 北京林业大学学报(社会科学 版), 14(4), 17-22.]

Wu HF, Rao J (2009) The influence of feminization of agriculture on agricultural development. Journal of Agrotechnical Economics, 2(3), 55-61. (in Chinese) [ 吴慧芳, 饶静 (2009) 农业女性化对农业发展的影响. 农业技术经济, 2(3), 55-61.]

Xue DY, Wu JY, Zhao FW (2012) Actions, progress and prospects in implementation of the Convention on Biological Diversity during the past 20 years in China. Biodiversity Science, 20, 623-632. (in Chinese with English abstract) [薛 达元, 武建勇, 赵富伟 (2012) 中国履行《生物多样性公 约》二十年: 行动、进展与展望. 生物多样性, 20, 623-632.]

Zhang TL, Zhang M (1994) The present population of the Tibetan nationality in China. Social Sciences in China, 15(1), 46-65.

(责任编委: 卢宝荣 责任编辑: 黄祥忠) 